\title{
COVID-19: una crisis que requiere medidas de formación urgentes
}

\author{
COVID-19: a crisis that requires urgent training measures
}

\author{
Diego Andrés Díaz-Guio, ${ }^{*}$ * Jeniffer Ospina-Vélez, ${ }^{*}$ Alejandra Ricardo-Zapata*
}

Estimada editora:

E n diciembre de 2019, en Wuhan, capital de la provincia de Hubei, en China, se reportó la aparición de un grupo de pacientes con síntomas como fiebre, progresión hacia distrés respiratorio agudo y cambios radiológicos compatibles con neumonía. En dicho grupo se aisló un nuevo coronavirus del grupo $\beta$ al que se le denominó SARS-CoV-2. El 11 de febrero de 2020, a esta enfermedad producida por este nuevo virus, la Organización Mundial de la Salud (OMS) le dio el nombre oficial de COVID-19 (acrónimo del inglés coronavirus disease), ${ }^{1,2}$ constituyendo el séptimo de una familia de coronavirus que puede infectar al humano.

Al 17 de marzo de 2020, COVID-19 está presente en 155 países, ha infectado a cerca de 200 mil personas y ha generado más de ocho mil muertes, siendo ya considerada una pandemia por la OMS. En América Latina se han reportado casos en la mayoría de los países, principalmente en Brasil, Chile, Perú, Ecuador y Panamá. ${ }^{3}$

El número reproductivo básico calculado para este virus es de aproximadamente 2-3, lo que le confiere un alto potencial de contagio, tal como se ha visto en China y más recientemente en Italia y España, lo que está logrando desbordar la capacidad de los sistemas de salud. ${ }^{4}$ Ante estos datos se han establecido políticas que apuntan al distanciamiento social, incluido el cese de actividades de formación presencial; medidas que buscan enlentecer el contagio. ${ }^{5}$

La atención de esta crisis requiere una gran cantidad de profesionales de ciencias de la salud, principalmente médicos, enfermeras y terapeutas respiratorios; estos profesionales deben estar formados en el manejo de la falla respiratoria aguda y el uso de equipos de protección personal (EPP) para evitar su propio contagio, ${ }^{6,7}$ ya que la contaminación en las áreas de tratamiento es muy alta. ${ }^{8}$
Teniendo en cuenta que la curva de reporte de casos confirmados en el mundo al día de hoy tiene una tendencia vertical, es decir, surgen una gran cantidad de pacientes enfermos al mismo tiempo, se deben gestionar rápidamente recursos como las unidades de cuidado intensivo, los ventiladores mecánicos, el personal entrenado y los equipos de protección personal. ${ }^{9}$ Un problema aun más grande es que estos recursos son limitados y más aún en regiones como la nuestra. Por esta razón es que debemos tomar medidas tempranas, como la creación de áreas de hospitalización a manera de unidades de cuidado intensivo extendidas y con profesionales no intensivistas, posiblemente residentes, internos de medicina y enfermería. Sin embargo, estas personas tienen un mayor riesgo de contagio por la diferencia en la formación en patologías infecciosas y menor experiencia en uso de los equipos de protección personal. ${ }^{10,11}$

Nos encontramos entonces en una paradoja: por un lado, debemos suspender las clases, cerrar los centros de simulación y promover el distanciamiento social efectivo y, por la otra, movilizar recursos y formar personal de salud. Una salida viable puede ser la educación virtual en cuestiones teóricas y tiempo corto con medidas especiales en los centros de simulación para los aspectos que obligatoriamente requieren experiencia, como lo es el uso de los equipos de protección personal.

En nuestro centro de simulación cambiamos las clases presenciales por clases virtuales mediante la plataforma Zoom ${ }^{\circledR}$, cuyos temas están relacionados con el manejo de COVID-19, haciendo énfasis en el manejo seguro de la vía aérea, sobre todo a los estudiantes de séptimo y octavo semestre de medicina; esta actividad tuvo una duración de 2.5 horas con cada semestre. Al final de estas clases se les aplicó una encuesta mediante un formulario de Google respecto a la percepción de calidad de las actividades en línea con una escala numérica de 1-5, además se 
realizaron preguntas abiertas en relación con las fortalezas y debilidades de la encuesta.

En esta actividad participaron 75 estudiantes, el $100 \%$ respondió la encuesta, el promedio de edad fue 22 años ( \pm 3 años) y la distribución por sexo fue de $54 \%$ para mujeres, el $52 \%$ pertenecía al séptimo semestre. El promedio de puntuación de la calidad de las actividades en línea fue de 4.25 y de la actividad realizada en Zoom de 4.59 .

Las respuestas referentes a las fortalezas se agruparon en cinco categorías según el análisis temático (Figura 1): no exposición a COVID-19, no perder clases, aprendizaje, comodidad e interacción en tiempo real; las debilidades se dividieron en tres categorías (Figura 2): hace falta la simulación clínica, dependencia de la actividad al internet y ninguna debilidad.

Para los profesionales de la salud de nuestra ciudad que enfrentarán la emergencia en primera línea (Urgencias-UCI), hicimos un taller de uso correcto del equipo de protección personal (donning/doffing) (Figura 3); esta actividad fue presencial y de una duración de tres horas. En dicho taller se revisó el estado de la cuestión de COVID-19, se practicó el uso seguro de los EPP y se midió la carga cognitiva antes y después de la intervención; los resultados de esta actividad se encuentran en proceso de publicación.

Figura 1:

Fortalezas de una actividad formativa en linea.

Figura 2:

Debilidades de una actividad formativa en linea.
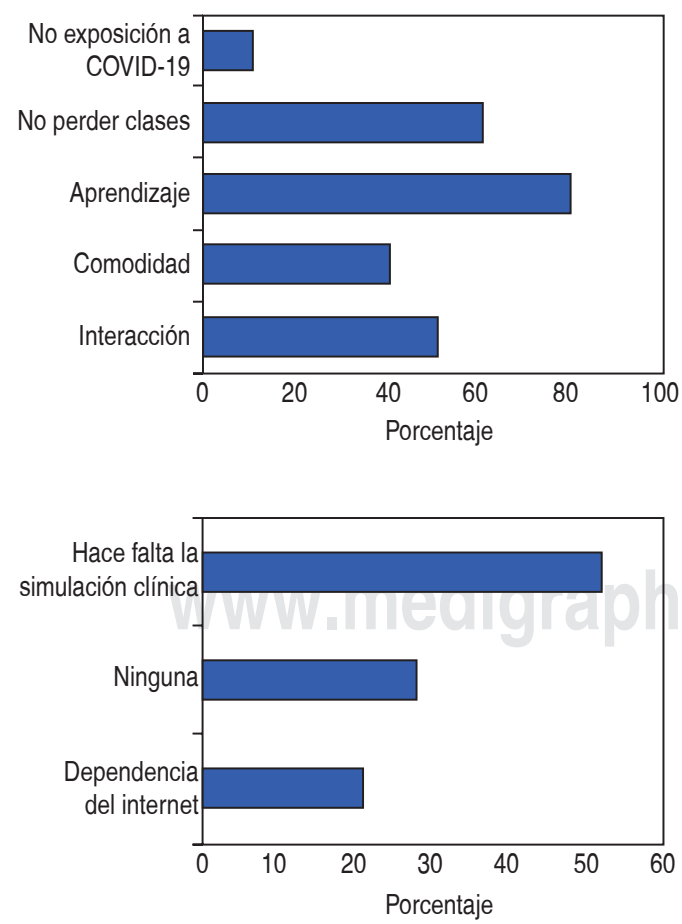

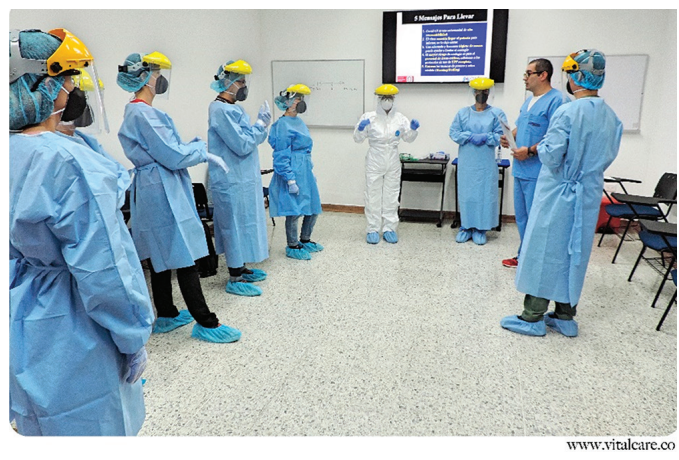

Figura 3: Taller de donning y doffing del equipo de protección personal.

La intención de esas actividades es clara: necesitamos formar personal para manejar a los pacientes más graves sin poner en riesgo su propia seguridad; así, realizar actividades académicas en línea mediante diferentes plataformas pueden ser de utilidad en momentos difíciles como los que hemos empezado a vivir; además son seguras, cómodas y permiten la interacción en tiempo real y mantener el ritmo de aprendizaje.

Un hecho indudable es que la curva de reporte de infectados en Latinoamérica va en ascenso, por ello, los centros de simulación podemos aportar por medio de la formación de nuestros profesionales en técnicas de uso seguro de los equipos de protección personal durante el proceso de atención de pacientes con COVID-19, esto puede llegar a ser de gran impacto en la práctica real cuando los números de esta región se parezcan a los asiáticos o europeos, iel momento de actuar es ahora!

\section{REFERENCIAS}

1. Lipsitch M, Swerdlow DL, Finelli L. Defining the epidemiology of COVID-19-Studies needed. N Engl J Med. 2020. doi: 10.1056/NEJMp2002125.

2. Chen X, Tian J, Li G, Li G. Comment Initiation of a new infection control system for the COVID-19 outbreak. Lancet Infect Dis. 2020; 3099 (20). Disponible en: http://dx.doi.org/10.1016/S1473-3099(20)30110-9.

3. World Health Organization. The Coronavirus Disease 2019 (COVID-19) Situation Report-57 [Internet]. 2020 [visitado el 17 de marzo de 2020]. Disponible en: https://www.who.int/docs/defaultsource/coronaviruse/situation-reports/20200317sitrep-57-COVID-19.pdf?sfvrsn=a26922f2_2

4. Cheng ZJ, Shan J. 2019 Novel coronavirus: where we are and what we know. Infection. 2020 Feb 18. doi: 10.1007/s15010-020-01401-y [Epub ahead of print].

5. Stevens $\mathrm{H}$. Why outbreaks like coronavirus spread exponentially, and how to "flatten the curve." The 
Washington Post [Internet]. 2020. Disponible en: https://www.washingtonpost.com/graphics/2020/ world/corona-simulator/

6. Wax RS, Christian MD. Practical recommendations for critical care and anesthesiology teams caring for novel coronavirus (2019-nCoV) patients. Can J Anaesth. 2020. doi: 10.1007/s12630-020-01591-x.

7. Verbeek JH, Rajamaki B, ljaz S, Tikka C, Ruotsalainen $\mathrm{JH}$, Edmond MB, et al. Personal protective equipment for preventing highly infectious diseases due to exposure to contaminated body fluids in healthcare staff. Cochrane Database Syst Rev. 2019; 7: CD011621.

8. Ong SWX, Tan YK, Chia PY, Lee TH, Ng OT, Wong MSY, et al. Air, surface environmental, and persona protective equipment contamination by severe acute respiratory syndrome coronavirus 2 (SARS-CoV-2) from a symptomatic patient. JAMA. 2020 Mar 4. doi: 10.1001/jama.2020.3227 [Epub ahead of print].

9. Halpern NA, Sloan M, Cancer K, Tan KS, Biostatistician AA, Sloan M, et al. US ICU resource availability for
COVID-19 [Internet Blog]. Society of Critical Care Medicine. 2020. Disponible en: https://sccm.org/ getattachment/Blog/March-2020/United-StatesResource-Availability-for-COVID-19/United-StatesResource-Availability-for-COVID-19.pdf?lang=en-US

10. Wang X, Pan Z, Cheng Z. Association between 2019nCoV transmission and N95 respirator use. J Hosp Infect. 2020 Mar 3. pii: S0195-6701(20)30097-9. doi: 10.1016/j.jhin.2020.02.021 [Epub ahead of print].

11. Huh S. How to train the health personnel for protecting themselves from novel coronavirus (COVID-19) infection during their patient or suspected case care. J Educ Eval Health Prof. 2020; 17: 10. doi: 10.3352/ jeehp.2020.17.10.

Correspondencia:

Diego Andrés Díaz-Guio

E-mail: andres.diaz@vitalcare.co 